\title{
An X-Band Dual-Polarized Vivaldi Antenna with High Isolation
}

\author{
Denghui Huang, ${ }^{1}$ Hu Yang, ${ }^{1}$ Yuqing Wu, ${ }^{2}$ and Fei Zhao ${ }^{3}$ \\ ${ }^{1}$ College of Electronic Science and Technology, National University of Defense Technology, Hunan 410073, China \\ ${ }^{2}$ Cavendish Laboratory, University of Cambridge, London, UK \\ ${ }^{3}$ The Southwest Electronics and Telecommunication Technology Research Institute, Chengdu 610041, China
}

Correspondence should be addressed to Denghui Huang; hdh15040004@163.com

Received 14 May 2017; Revised 14 July 2017; Accepted 20 July 2017; Published 24 August 2017

Academic Editor: Luciano Tarricone

Copyright (C) 2017 Denghui Huang et al. This is an open access article distributed under the Creative Commons Attribution License, which permits unrestricted use, distribution, and reproduction in any medium, provided the original work is properly cited.

\begin{abstract}
An X-band dual-polarized Vivaldi antenna with high isolation is proposed. The procedure of this antenna design includes the conventional Vivaldi antenna with regular slot edge (RSE), the dual-polarized Vivaldi antenna with two Vivaldi antennas which have different feeding point positions in a cross-shaped form, and the two Vivaldi antennas with a galvanic contact in soldering point. By applying the RSE, it reduced the dimensions of the Vivaldi antenna and improved its radiation performance. The modified antenna is fabricated and measured. The measured results show that $S 11<-10 \mathrm{~dB}$ at the entire X-band for the two Vivaldi antennas. The isolation (S21) between the two feeding ports, which has been improved by applying the different feeding point positions and the galvanic contact in soldering point, is better than $34 \mathrm{~dB}$ at X-band. In addition, the cross-polarized discrimination is better than $21 \mathrm{~dB}$ for the two Vivaldi antennas, and the measured results also include the gain of two Vivaldi antennas.
\end{abstract}

\section{Introduction}

The Vivaldi antenna was firstly described by Gibson in 1979 [1] and then developed in an antipodal shape [2]. The Vivaldi antenna has been considered for many radar or communication system applications because it is an ultrawideband device, it has a planar structure, and it is lightweight, of high efficiency, and of high gain. For conventional Vivaldi antennas, the study results show that the electric field vector of the Vivaldi antenna is parallel to the substrate, which is single-linear-polarized on its two major radiation planes.

In some modern high-data or very high resolution imaging radar systems, the application of polarization diversity can enhance the efficiency of such systems because the polarization information delivers further information about the target characteristics. So in order to improve the performance of such systems, the antennas are required to have the characteristics of both ultrawideband and dual polarization. The dual-polarized Vivaldi antenna is a good choice which can be used in modern imaging radar systems at X-band. In recent years, many methods have been presented, to implement two orthogonal cases of polarization with Vivaldi antennas. As in [3], the dual-polarized antenna consists of four antipodal Vivaldi antennas and places them along the outer edge. And in [4-6], the dual-polarized Vivaldi antenna array has been presented, and the conventional Vivaldi antenna or all-metal Vivaldi antenna was used as the array element.

In [7-11], the dual-polarized Vivaldi antenna is composed of two conventional Vivaldi antennas in a cross-shaped form without galvanic contact, and the isolation $(S 21)$ between the two feeding ports is $23 \mathrm{~dB}$ in [9] and $25 \mathrm{~dB}$ in $[7,10]$. The best result is presented in [12], the isolation is better than $30 \mathrm{~dB}$ at the entire function frequency band, and for the two Vivaldi antennas, one is ahead of the other, which avoids the antenna feeding lines overlapping each other. Additionally, the published antennas in [7-11] cannot be used as the dual-polarized antenna array element because the width of antenna is larger than a wavelength corresponding to the higher frequency of the operating band, which will lead to antenna array with grating lobe in higher frequency band.

In this paper, a modified dual-polarized Vivaldi antenna with good radiation performance and high isolation is designed and measured. On the one hand, the low cut-off frequency of the Vivaldi antenna was extended by RSE which 


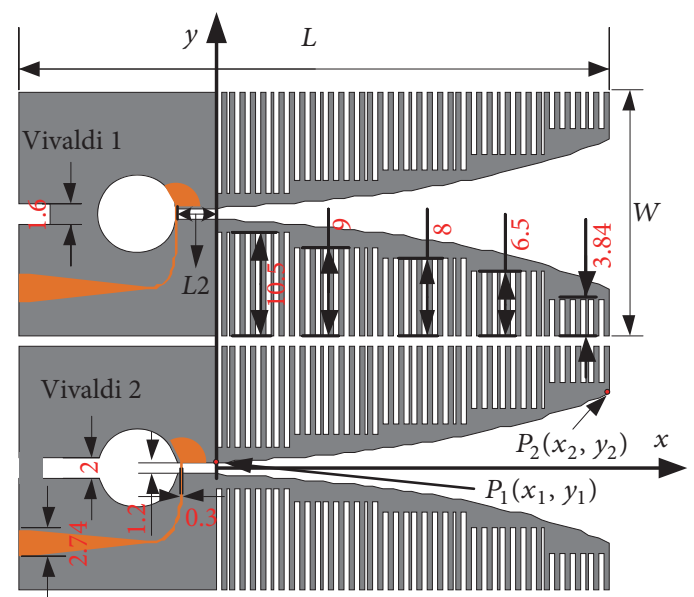

FIGURE 1: Geometry of the designed antenna elements: Vivaldi 1 and Vivaldi 2. Dimensions are in $\mathrm{mm}$.

indicates that it can be used as the dual-polarized antenna array element at X-band, and the radiation performance was also improved. On the other hand, we used the different feeding point positions for two Vivaldi antennas instead of the one Vivaldi antenna being ahead of another Vivaldi antenna, and there is a galvanic contact in soldering point between the two Vivaldi antennas. The overall effect is that the high isolation $(S 21)$ is better than $34 \mathrm{~dB}$. Additionally, for the two Vivaldi antennas, the measured gain is between $6.4 \mathrm{~dB}$ and $12.2 \mathrm{~dB}$ at X-band.

\section{Antenna Design}

Figure 1 demonstrates the geometry of the dual-polarized Vivaldi antenna elements: Vivaldi 1 and Vivaldi 2. Those antennas with the same RSE are fed by a $2.74 \mathrm{~mm}$ microstrip line, and the characteristic impedance of microstrip line can be calculated as $50 \Omega$ in order to match with SMA connector. The thickness of the substrate is $1 \mathrm{~mm}$ and its relative permittivity is 2.65 . The substrate dimension $\left(L_{\mathrm{SUB}} \times\right.$ $W_{\text {SUB }}$ ) is $60 \times 25 \mathrm{~mm}^{2}$.

The inside of the radiation flares for Vivaldi 1 and Vivaldi 2 fits the following exponential curves:

$$
\begin{aligned}
y & =C_{1} \times e^{\left(\alpha_{1} \times x\right)}+C_{2}, \\
C_{1} & =\frac{y_{1}-y_{2}}{e^{\left(\alpha_{1} \times x_{1}\right)}-e^{\left(\alpha_{1} \times x_{2}\right)},} \\
C_{2} & =\frac{y_{2} e^{\left(\alpha_{1} \times x_{1}\right)}-y_{1} e^{\left(\alpha_{1} \times x_{2}\right)}}{e^{\left(\alpha_{1} \times x_{1}\right)}-e^{\left(\alpha_{1} \times x_{2}\right)}} .
\end{aligned}
$$

$\alpha_{1}=0.052$ stands for exponential rate. $P_{1}=(0,0.6)$ and $P_{2}=(40,7.6)$ stand for the start and end point of exponential curves.

According to [12], for the conventional Vivaldi antenna, the radius of the stub limits the upper working frequency and in order to get the best impedance matching, the cavity should keep the same radius as the stub. In [12], it offers a fast way to design wideband Vivaldi antenna except that it takes
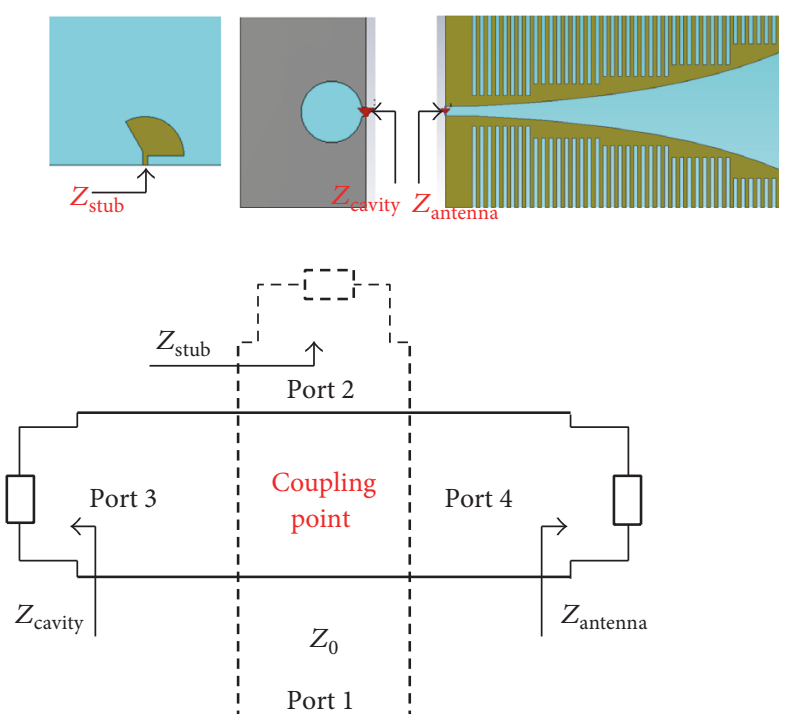

ニニニ Microstrip line

- Slot line

FIGURE 2: The network for the equivalent circuit of Vivaldi antenna.

the position of the feeding or coupling point into account. In this paper, based on the theory of the equivalent circuit of Vivaldi antenna [13], the position of the feeding point has been studied.

The network for the equivalent circuit model of the Vivaldi antenna is shown in Figure 2. From the coupling point of microstrip to slot-line transmission, the Vivaldi antenna can be equivalent to a microwave network with four ports. As shown in Figure 2, $Z_{0}$ is the character impedance of the microstrip line and $Z_{\text {stub }}, Z_{\text {cavity }}$ denote the input impedance of the microstrip open-end and slot short-end, respectively. $Z_{\text {antenna }}$ expresses the input impedance of the isolated Vivaldi antenna fed by a slot transmission line from the coupling point.

Based on [13], the input reflection coefficient $\Gamma_{\text {in }}$ for port 1 can be given:

$$
\Gamma_{\text {in }}=\frac{Z_{l}-Z_{0}}{Z_{l}+Z_{0}}
$$

where

$$
\begin{aligned}
Z_{l}= & n^{2} \times \operatorname{Re}\left(\frac{Z_{\text {antenna }} \times Z_{\text {cavity }}}{Z_{\text {antenna }}+Z_{\text {cavity }}}\right)+j \times n \\
& \times \operatorname{Im}\left(\frac{Z_{\text {antenna }} \times Z_{\text {cavity }}}{Z_{\text {antenna }}+Z_{\text {cavity }}}\right)+Z_{\text {stub }}
\end{aligned}
$$

and the coupling coefficient $n$ can be found in [14].

For the different feeding or coupling point position $L 2$, shown in Figure 1, the real and imaginary parts of $Z_{l}$ can be calculated with the simulated input impedance for open-end stub, $Z_{\text {stub }}$, short-end cavity, $Z_{\text {cavity }}$, and the isolated Vivaldi antenna fed by a slot transmission line, $Z_{\text {antenna }}$, shown in Figure 2, and those simulations were also presented in [12]. 

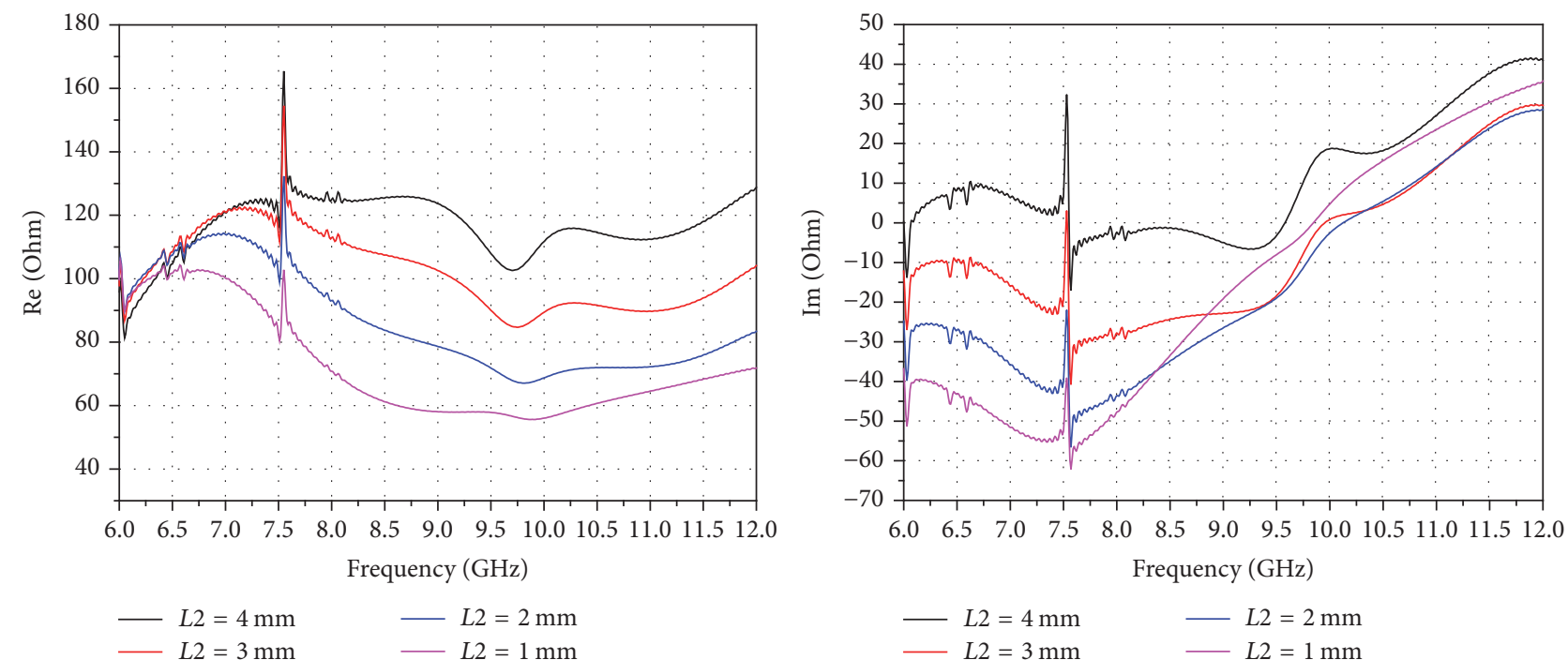

Figure 3: The real and imaginary parts of the $Z_{l}$.

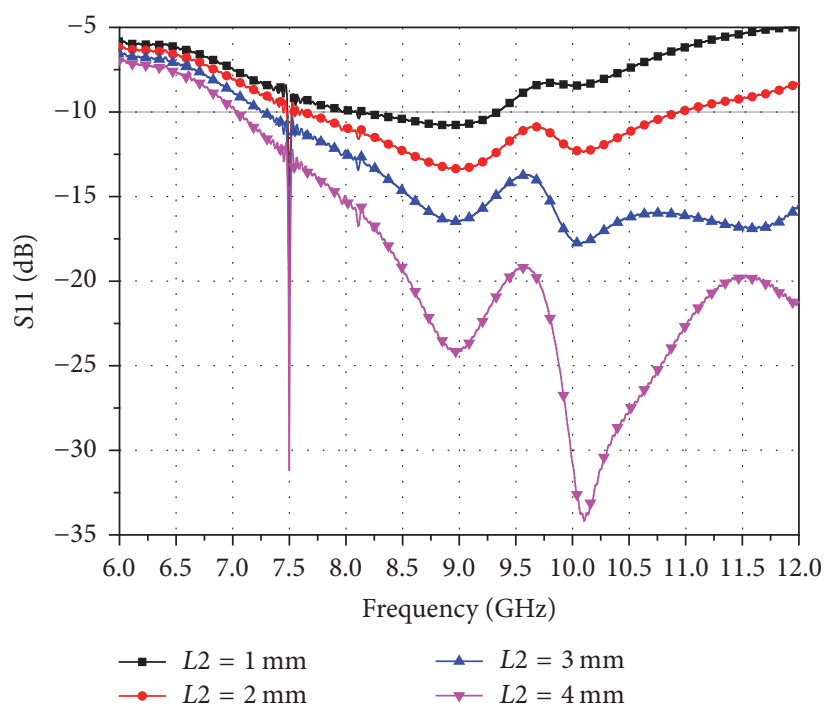

FIGURE 4: Simulated S11 for the different feeding point positions.

Figure 3 shows the real and imaginary parts of $Z_{l}$ with different $L 2$. As the feeding point is closer to the cavity, the smaller variation of the imaginary and real parts of the $Z_{l}$ has been gotten, which indicates that the wider bandwidth for Vivaldi antenna will be obtained. Based on the full-wave electromagnetic model simulation of Vivaldi antenna with different $L 2$, the magnitude of the reflection coefficient with different feeding point position $L 2$ is shown in Figure 4; the simulated results show that the feeding point position is closer to the cavity; the wider frequency band has been gotten, which is in good agreement with the conclusion shown in Figure 3.

Based on the study of the feeding point position, the Vivaldi antenna element for dual-polarized Vivaldi antenna has been designed. The coupling point $L 2$ is greater than
$3 \mathrm{~mm}$ for $\mathrm{S} 11<-10 \mathrm{~dB}$ at X-band and the optimal cavity radius is $4 \mathrm{~mm}$, and the radius of the stub is $2.5 \mathrm{~mm}$, with a stub angle of $120^{\circ}$. Moreover, the simulated $S 11$ and gain of Vivaldi antenna with and without RSE are shown in Figure 5. In Figure 5(a), the low cut-off frequency is extended by RSE from $9.6 \mathrm{GHz}$ to $7 \mathrm{GHz}$ without increasing the volume of the antenna, and the gain of Vivaldi antenna is also improved by RSE at X-band in Figure 5(b).

For the most common dual-polarized Vivaldi antenna shown in Figure 6(a), in order to avoid the feeding lines overlapping each other, Vivaldi 2 is ahead of Vivaldi 1 with $d \mathrm{~mm}$. Based on the parameter study of $d$, which is the distance between Vivaldi 2 and Vivaldi 1, the isolation between two antenna ports is shown in Figure 7(a). The simulated results indicate that the isolation between two ports 


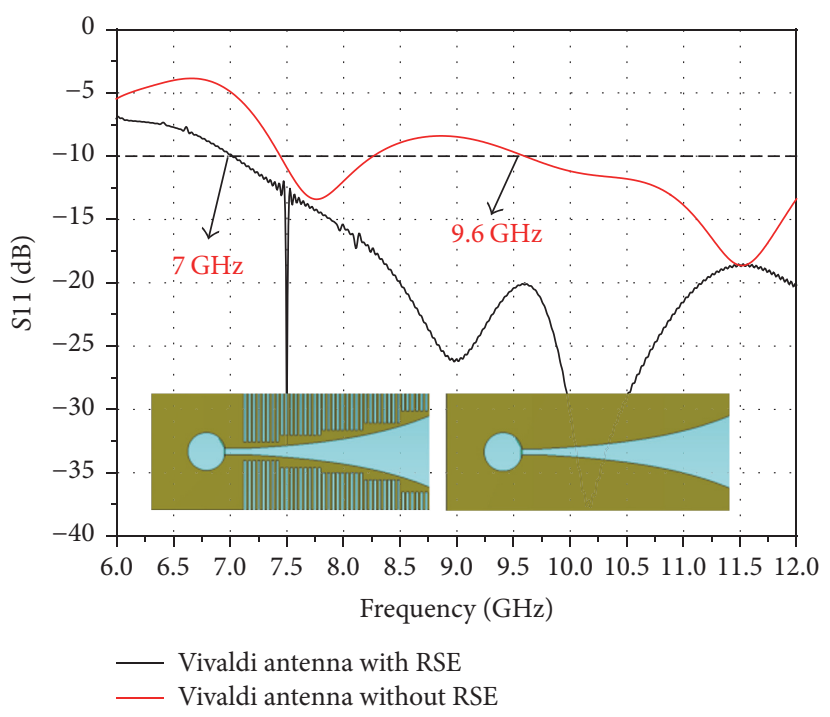

(a)

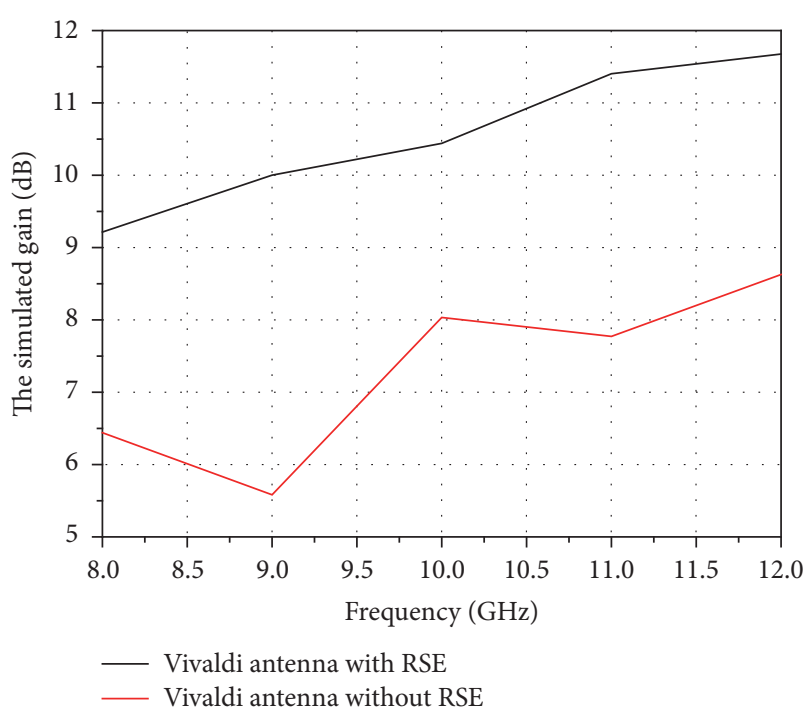

(b)

FIGURE 5: The simulated S11 and gain for Vivaldi antenna with RSE and Vivaldi antenna without RSE.

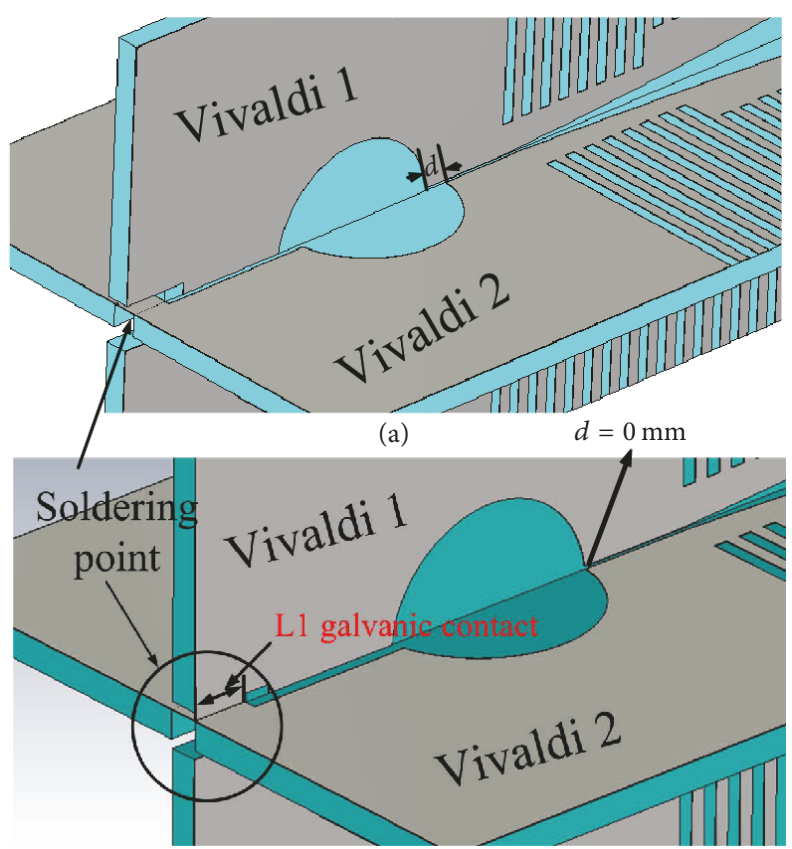

(b)

Figure 6: The structure of dual-polarized Vivaldi antenna: (a) conventional dual-polarized Vivaldi antenna; (b) modified dual-polarized Vivaldi antenna.

is better than $24 \mathrm{~dB}$ for $d=2 \mathrm{~mm}$, while it is better than $29 \mathrm{~dB}$ for $d=1 \mathrm{~mm}$. As shown in Figure 6(b), we used the different feeding or coupling point position $L 2$ for the two Vivaldi antenna elements instead of Vivaldi 1 being ahead of Vivaldi 2, which avoids the feeding lines overlapping each other. $L 2$ for Vivaldi 1 is $4.1 \mathrm{~mm}$, while $L 2$ is $3.5 \mathrm{~mm}$ for Vivaldi 2. The isolation between the two feeding ports is better than $32 \mathrm{~dB}$ with $d=0 \mathrm{~mm}$.
Figure 7(b) plots the isolation between the two feeding ports with galvanic contact in soldering port shown in Figure 6(b). The length of galvanic contact $L 1$ has an effect on the return loss of Vivaldi 2, and the lower frequency limit is $8.3 \mathrm{GHz}$ when $L 1$ exceeds $3 \mathrm{~mm}$. In this antenna design, in order to complement dual polarization, we place two Vivaldi antennas cross-shaped with different feeding point position, which avoids the feeding lines overlapping each other, and 


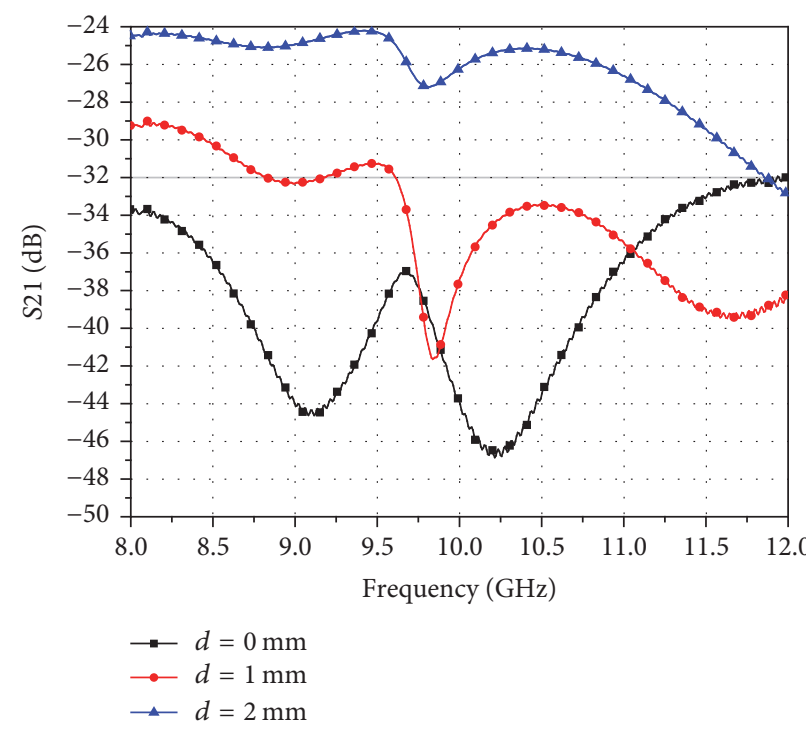

(a)

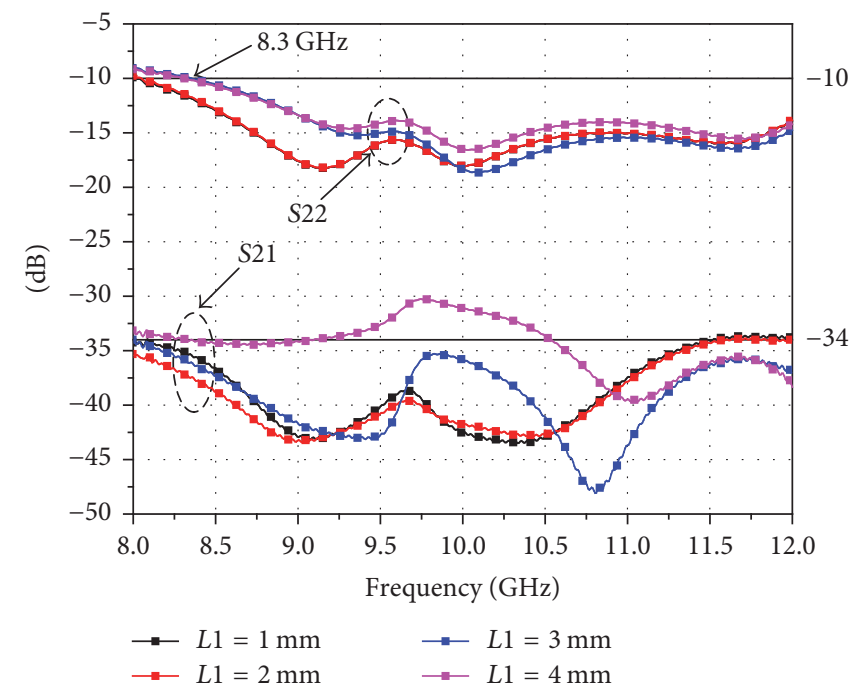

(b)

Figure 7: (a) Effect of the different $d$ on the isolation of two ports. (b) Simulated return loss of Vivaldi 2 and isolation with the different length of galvanic contact.

also there is a galvanic contact in soldering point. The overall effect is that the return loss of the two Vivaldi antennas is lower than $-10 \mathrm{~dB}$ at $\mathrm{X}$-band, and the isolation between the two feeding ports is better than $34 \mathrm{~dB}$ with $L 1=2 \mathrm{~mm}$ galvanic contact.

\section{Results and Discussion}

The photograph of modified dual-polarized Vivaldi antenna has been fabricated and measured. Figure 8(a) shows the top and bottom view of the constructed Vivaldi 1 and Vivaldi 2. By using the different feeding point positions, the lengths of the slots in Vivaldi 1 and Vivaldi 2 are 16.2 and $43.8 \mathrm{~mm}$, and the summarized length of the slots is equal to the length of antenna, which indicates the $0 \mathrm{~mm}$ displacement between Vivaldi 1 and Vivaldi 2. The $0 \mathrm{~mm}$ displacement between Vivaldi 1 and Vivaldi 2 also indicates that the separate antennas almost have the same phase center in $x$ axis although the feeding point of Vivaldi 2 ahead of Vivaldi 1 is $0.6 \mathrm{~mm}$. The width of the slots is $1.1 \mathrm{~mm}$ and it is wider than the thickness of the substrate $(1 \mathrm{~mm})$, where Vivaldi 1 can be inserted into Vivaldi 2 in a cross-shaped form. The structure of the modified dual-polarized Vivaldi antenna is shown in Figure 8(b). As shown in Figure 8(b), there is a galvanic contact in soldering point and the feeding point of Vivaldi 2 ahead of Vivaldi 1.

Figure 9 illustrates the measured $S$-parameters of the dual-polarized Vivaldi antenna. The $S$-parameters were measured with Agilent Performance Network Analyzer N5230C. As can be seen from Figure 9, the frequency band of $S 11<$ $-10 \mathrm{~dB}$ is $7.4-12 \mathrm{GHz}$ for Vivaldi 1 , while it is $7.8-12 \mathrm{GHz}$ for Vivaldi 2, where the frequency band for $S 11<-10 \mathrm{~dB}$ is covering the X-band. In addition, with the help of the different feeding point positions for Vivaldi antennas and the only galvanic contact in soldering point, the isolation between two ports is better than $34 \mathrm{~dB}$ at X-band. Compared to the simulated $S 11$ and $S 22$, because the losses of the dielectric and the SMA connector are not taken into account in simulations, the measured $S 11$ and $S 22$ are lower than the simulated results.

Figure 10 plots the measured radiation patterns of Vivaldi 1 and Vivaldi 2 at different frequencies for $E$-plane and $H$ plane. As shown in Figure 10, both antennas have endfire characteristics with the main lobe in the axial direction $(X$ direction in Figure 1). And for the two Vivaldi antennas, they have a similar performance within the X-band. Additionally, based on the measured results in Figure 10, the $E$-plane $3 \mathrm{~dB}$ beamwidth for Vivaldi 1 is $63^{\circ}$ at $8 \mathrm{GHz}, 56^{\circ}$ at $10 \mathrm{GHz}$, and $39^{\circ}$ at $12 \mathrm{GHz}$, while it is $67^{\circ}$ at $8 \mathrm{GHz}, 51^{\circ}$ at $10 \mathrm{GHz}$, and $35^{\circ}$ at $12 \mathrm{GHz}$ for Vivaldi 2. All the results indicate that Vivaldi 1 and Vivaldi 2 have a good radiation performance at the total frequency band.

According to the measured radiation patterns, the measured cross-polarization discrimination versus frequency of Vivaldi 1 and Vivaldi 2 is shown in Figure 11. For Vivaldi 1, the cross-polarization discrimination is below $21.9 \mathrm{~dB}$ at the $\mathrm{X}$-band and is below $21 \mathrm{~dB}$ for Vivaldi 2 . In addition, the dualpolarized Vivaldi antenna with good polarization purity can be applied to many radar systems at X-band.

The gain variation of Vivaldi 1 and Vivaldi 2 at the entire frequency range is shown in Figure 12. As shown in Figure 12, the gain of Vivaldi 1 is between $7.4 \mathrm{~dB}$ and $11.6 \mathrm{~dB}$ at the $\mathrm{X}$-band, while is between $6.4 \mathrm{~dB}$ and $12.2 \mathrm{~dB}$ at the same frequency band for Vivaldi 2. The gain of the antennas is improved with the increase of the frequency. Compared to Vivaldi 2, Vivaldi 1 has approximately the same gain from $9.2 \mathrm{GHz}$ to $12 \mathrm{GHz}$, and at $8 \mathrm{GHz}$, the gain of Vivaldi 1 is $1 \mathrm{~dB}$ higher than Vivaldi 2. The difference of the gain between 
Vivaldi 2
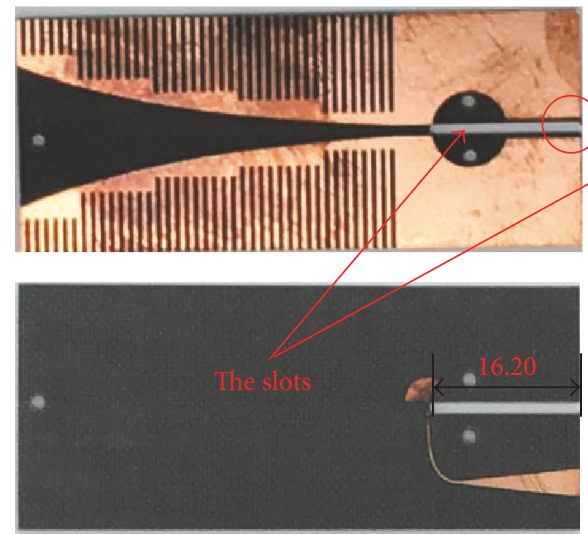

(a)

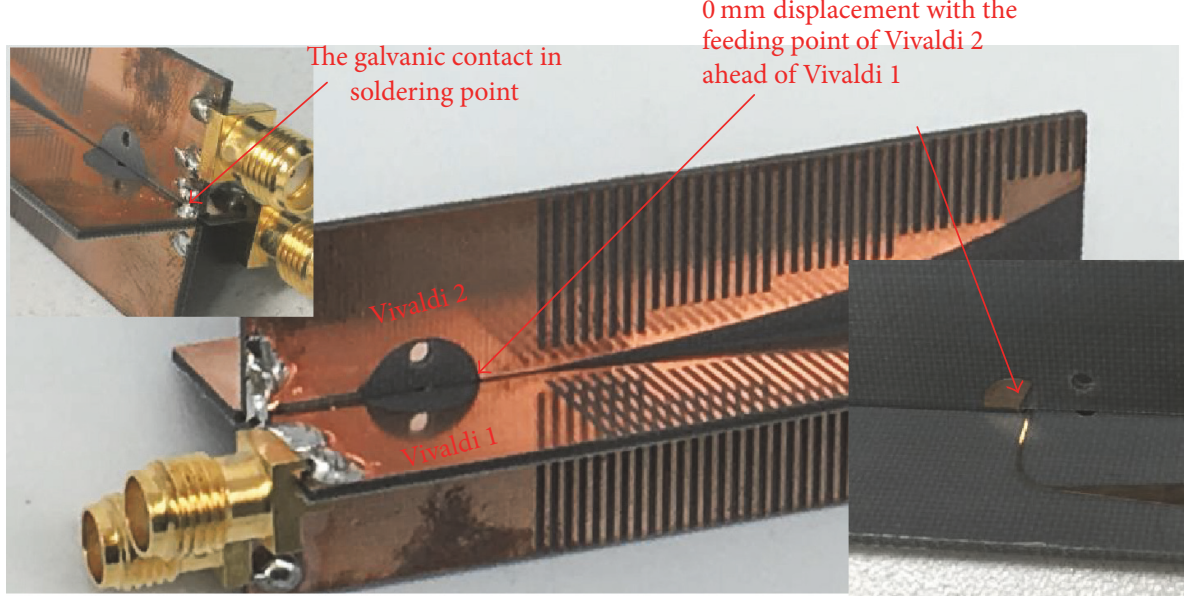

(b)
Vivaldi 1

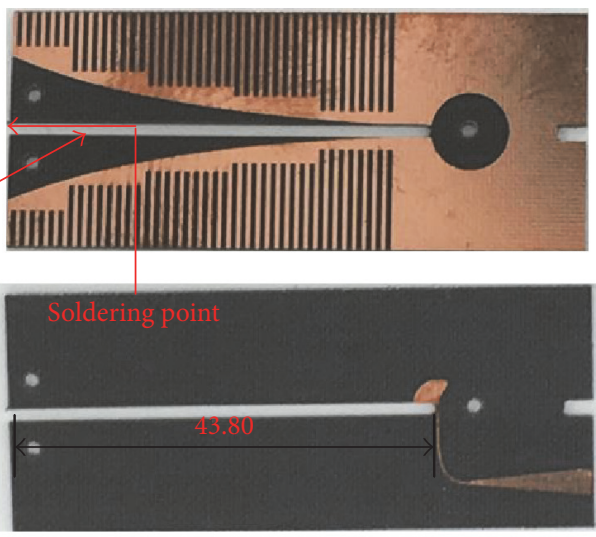

$0 \mathrm{~mm}$ displacement with the feeding point of Vivaldi 2 ahead of Vivaldi

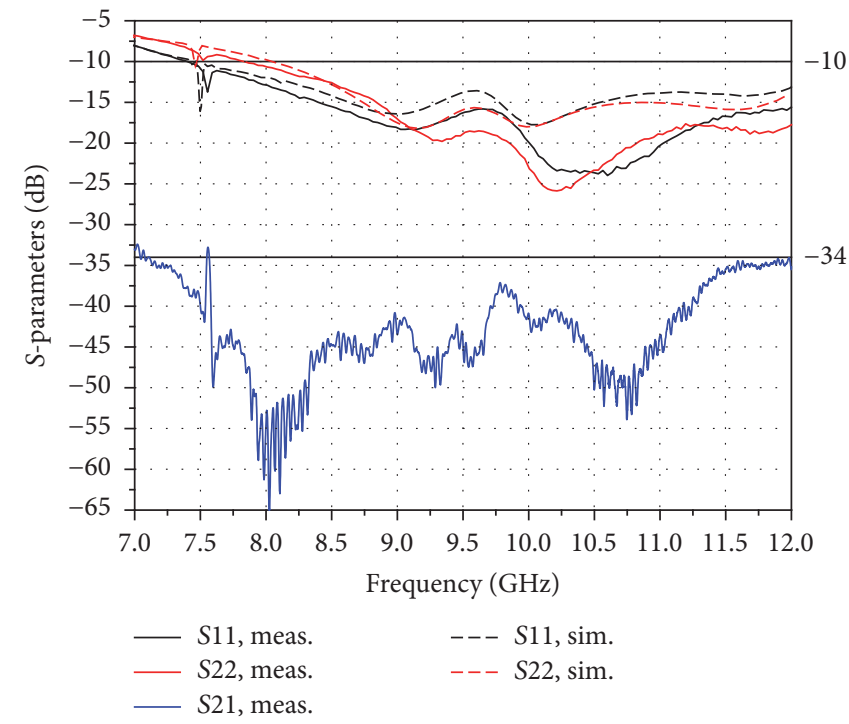

FIGURE 9: Simulated and measured S-parameters of the dual-polarized Vivaldi antenna. 

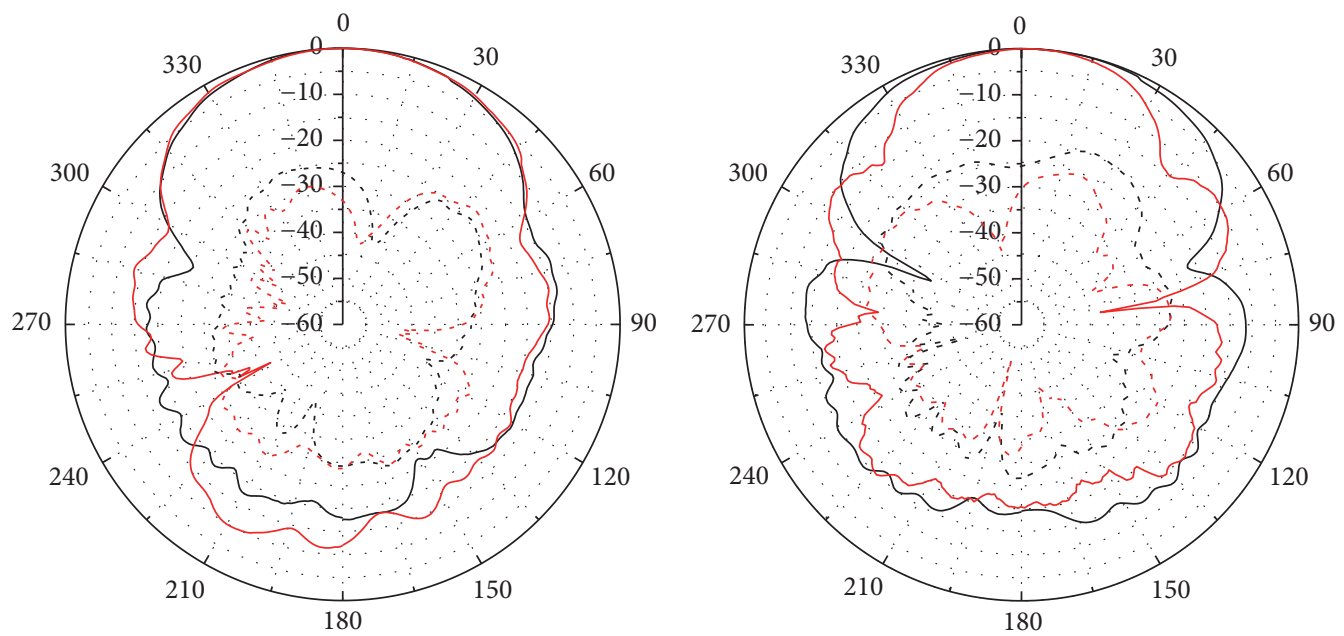

E-plane at $8 \mathrm{GHz}$

$H$-plane at $8 \mathrm{GHz}$

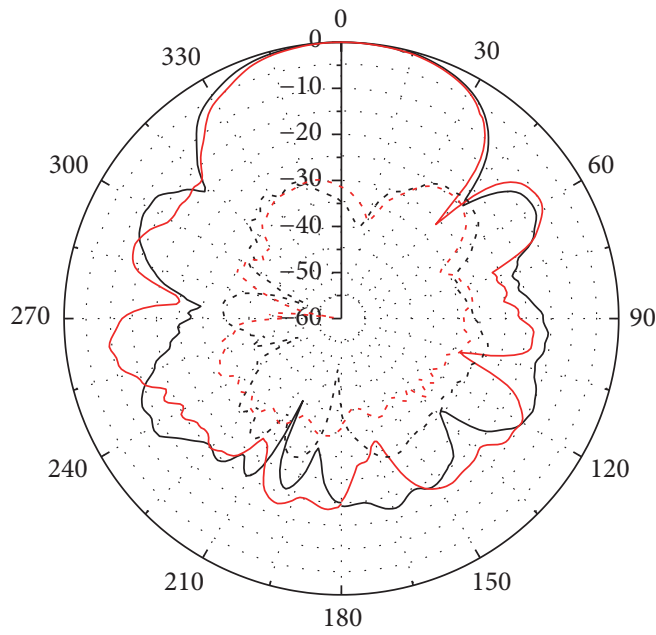

E-plane at $10 \mathrm{GHz}$

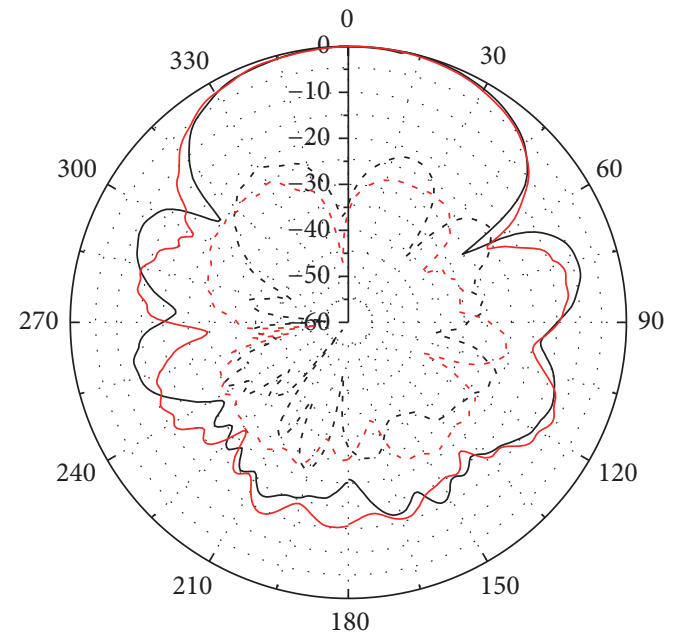

$H$-plane at $10 \mathrm{GHz}$
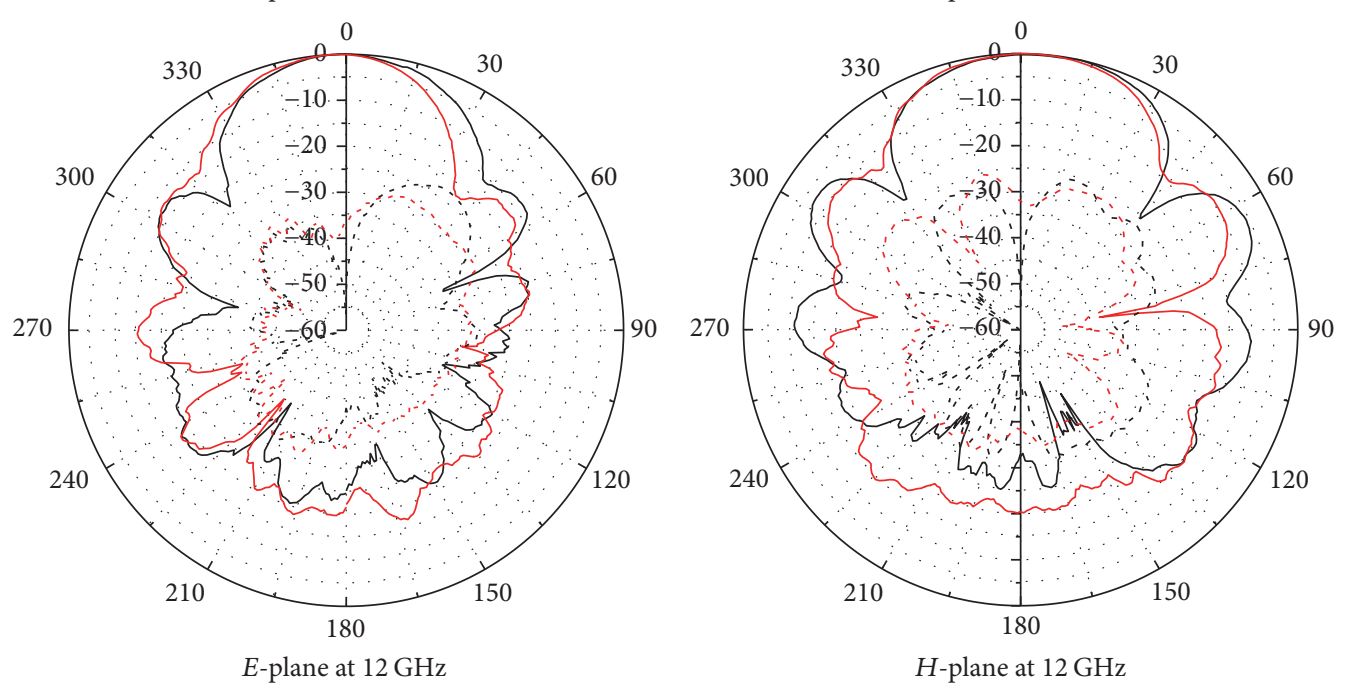

Figure 10: $E$ - and $H$-plane radiation patterns of the antennas at different frequencies with black solid lines for Vivaldi 1 copolarization, red solid line for Vivaldi 2 copolarization, black short dash line for Vivaldi 1 cross-polarization, and red short dash line for Vivaldi 2 crosspolarization. 


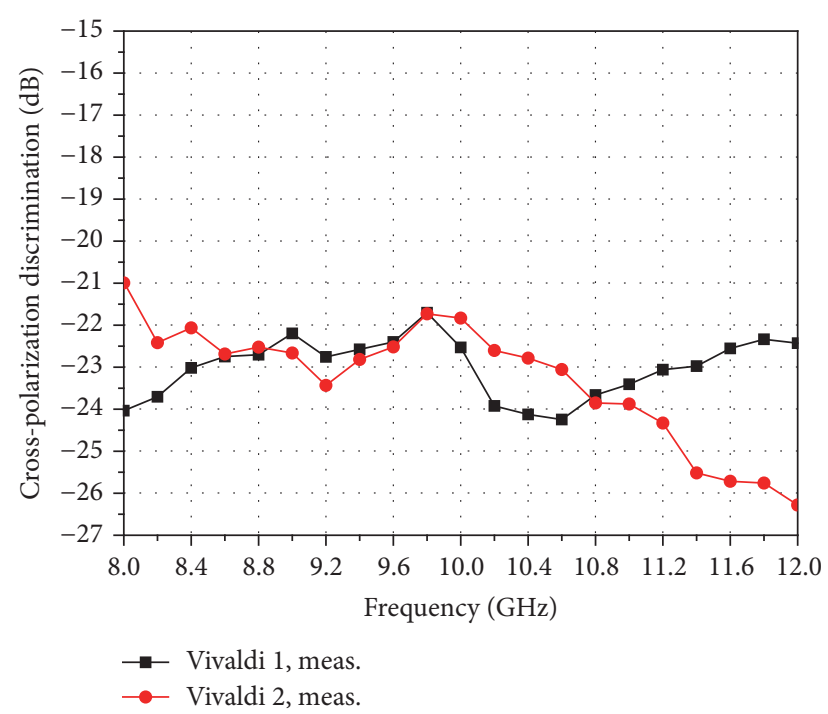

FIGURE 11: Variation of the cross-polarization discrimination for Vivaldi 1 and Vivaldi 2.

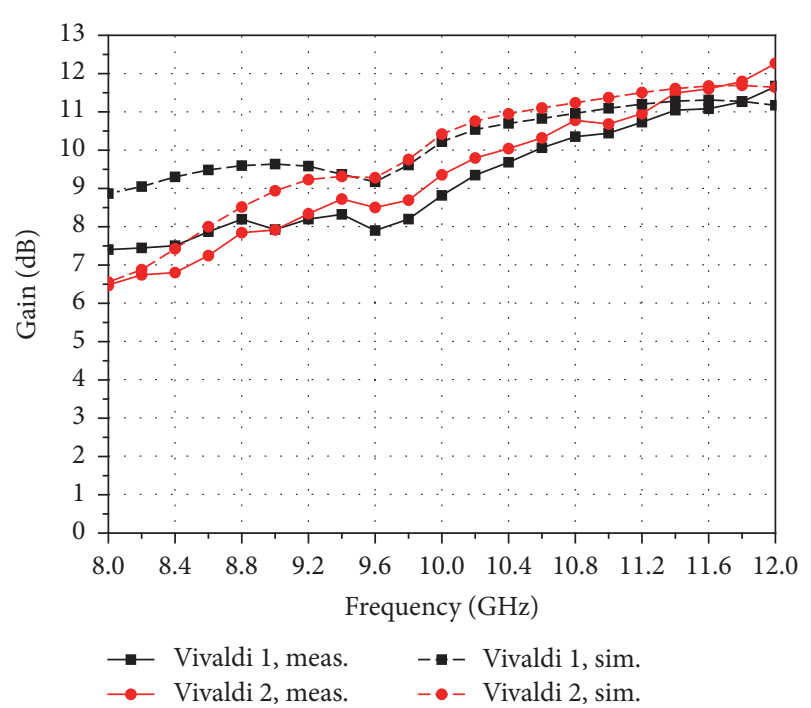

FIGURE 12: Simulated and measured gain for Vivaldi 1 and Vivaldi 2.

Vivaldi 1 and Vivaldi 2 in lower frequency is mainly caused by the slot behind Vivaldi 2 and the manufacturing error in the two Vivaldi antennas. In addition, those factors also caused the difference on the return loss for Vivaldi 1 and Vivaldi 2 shown in Figure 9. For the two Vivaldi antennas, the measured gain is lower than the simulations, because of the losses of the dielectric.

\section{Conclusion}

A modified X-band dual-polarized Vivaldi antenna has been designed and constructed. First, the dimensions of the dualpolarized Vivaldi antenna elements were reduced by RSE. And for the two Vivaldi antennas, the measurements show that the frequency band for $S 11<-10 \mathrm{~dB}$ is covering the X-band. With the help of the different feeding point positions and the only galvanic contact in soldering point, the high isolation between two antenna ports is better than $34 \mathrm{~dB}$. Additionally, the cross-polarization discrimination for Vivaldi 1 and Vivaldi 2 is below $21 \mathrm{~dB}$, and they both have approximately the same gain at the entire frequency band. Finally, all measurements which support our simulations indicate that the dual-polarized Vivaldi antenna has miniaturized dimensions, high isolation, good radiation performance, and good polarization purity, which can be applied in many imaging radar system applications at X-band.

\section{Conflicts of Interest}

The authors declare that they have no conflicts of interest.

\section{References}

[1] P. J. Gibson, "Vivaldi aerial," Conference Proceedings - European Microwave Conference, pp. 101-105, 1979.

[2] E. Gazit, "Improved design of the Vivaldi antenna," IEE Proceedings H: Microwaves, Antennas and Propagation, vol. 135, no. 2, pp. 89-92, 1988.

[3] H. Loui, J. P. Weem, and Z. Popović, "A dual-band dualpolarized nested vivaldi slot array with multilevel ground plane," IEEE Transactions on Antennas and Propagation, vol. 51, no. 9, pp. 2168-2175, 2003.

[4] S. Balling, M. Hein, M. Hennhofer, G. Sommerkorn, R. Stephan, and R. Thoma, "Broadband dual polarized antenna arrays for mobile communication applications," in Proceedings of the $33 \mathrm{rd}$ European Microwave Conference, 2003, pp. 927-930, Munich, Germany, October 2003.

[5] J. Remez, A. Segal, and R. Shansi, "Dual-polarized wideband widescan multibeam antenna system from tapered slotline elements array," IEEE Antennas and Wireless Propagation Letters, vol. 4, no. 1, pp. 293-296, 2005.

[6] J.-B. Yan, S. Gogineni, B. Camps-Raga, and J. Brozena, "A dualpolarized 2-18-GHz Vivaldi array for airborne radar measurements of snow," Institute of Electrical and Electronics Engineers. Transactions on Antennas and Propagation, vol. 64, no. 2, pp. 781-785, 2016.

[7] G. Adamiuk, R. T. Zwick, and W. Wiesbeck, "Dual-orthogonal polarized vivaldi antenna for ultra wideband applications," in Proceedings of the 17th International Conference on Microwaves, Radar and Wireless Communications (MIKON '08), pp. 1-4, May 2008.

[8] L. Song and Q. Fang, "Design and measurement of a kind of dual polarized Vivaldi antenna," in Proceedings of the 2011 Cross Strait Quad-Regional Radio Science and Wireless Technology Conference, CSQRWC 2011, pp. 494-497, chn, July 2011.

[9] X. Han, L. Juan, C. J. Cui, and Y. Lin, "UWB dual-polarized Vivaldi antenna with high gain," in Proceedings of the International Conference on Microwave and Millimeter Wave Technology (ICMMT '12), vol. 3, pp. 1-4, May 2012.

[10] L. Reichardt, J. Kowalewski, L. Zwirello, and T. Zwick, "Compact, Teflon embedded, dual-polarized ultra wideband (UWB) antenna," in Proceedings of the Joint 2012 IEEE International Symposium on Antennas and Propagation and USNC-URSI National Radio Science Meeting, APSURSI 2012, July 2012. 
[11] Y. Li, M. Su, Y. Z. Sheng, and L. Dong, "Ultra-wideband dual polarized probe for measurement application," in Proceedings of the in Proceedings of the International Symposium on Antennas Propagation, pp. 1025-1028, 2013.

[12] M. Sonkki, D. Sánchez-Escuderos, V. Hovinen, E. T. Salonen, and M. Ferrando-Bataller, "Wideband dual-polarized crossshaped Vivaldi antenna," IEEE Transactions on Antennas and Propagation, vol. 63, no. 6, pp. 2813-2819, 2015.

[13] C. Deng and Y. J. Xie, "Design of resistive loading Vivaldi antenna," IEEE Antennas and Wireless Propagation Letters, vol. 8, pp. 240-243, 2009.

[14] J. B. Knorr, "Slot-line transitions (short papers)," IEEE Transactions on Microwave Theory Techniques, vol. 22, pp. 548-554, 1974. 


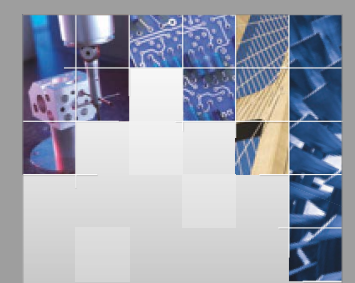

\section{Enfincering}
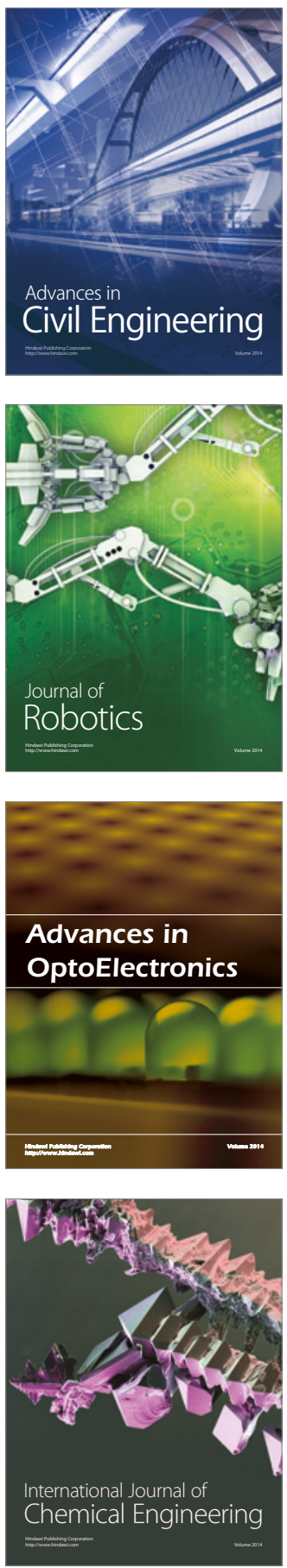

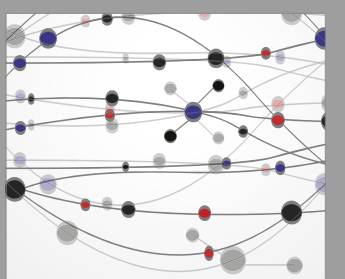

The Scientific World Journal

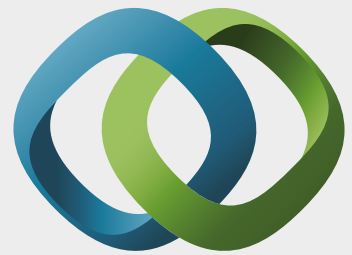

\section{Hindawi}

Submit your manuscripts at

https://www.hindawi.com
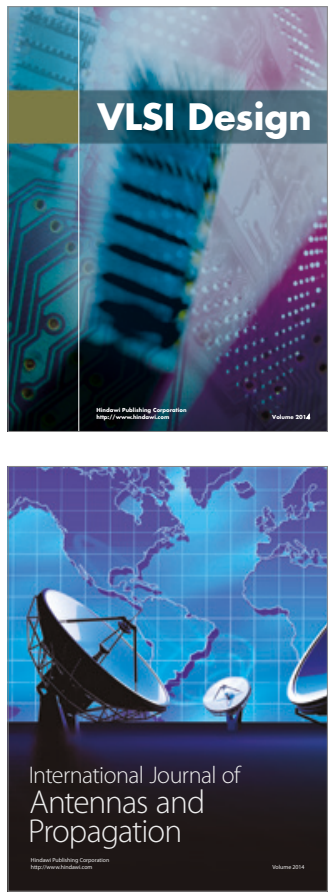

\section{Rotating}

Machinery
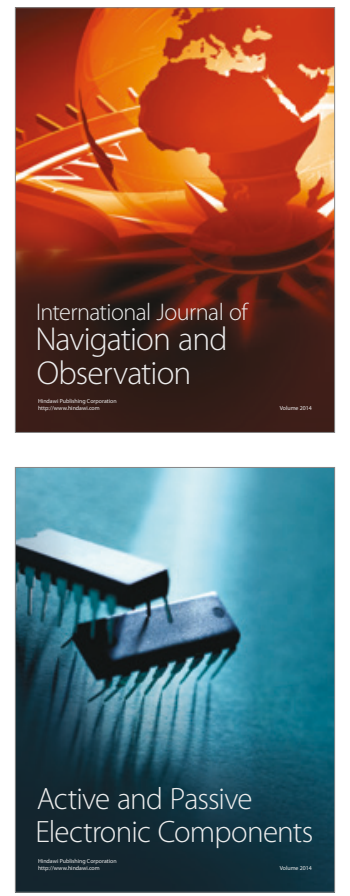
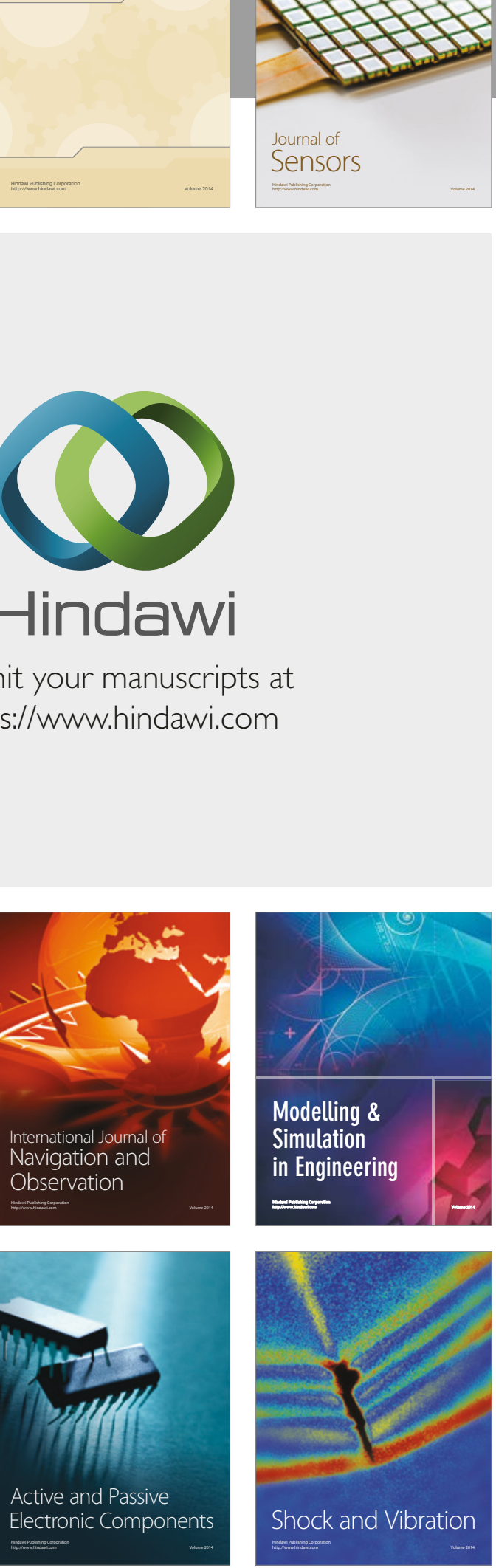
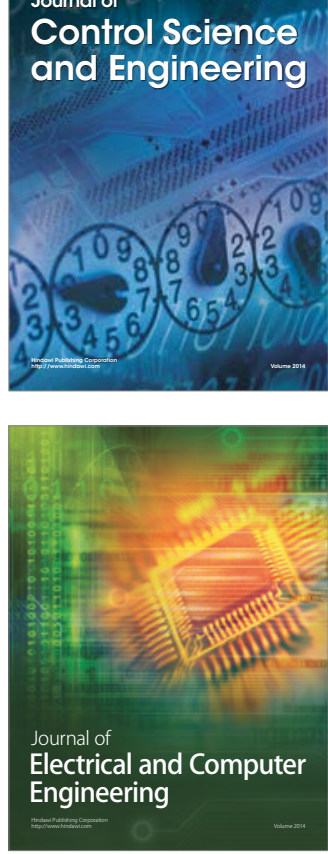

Distributed

Journal of

Control Science

and Engineering
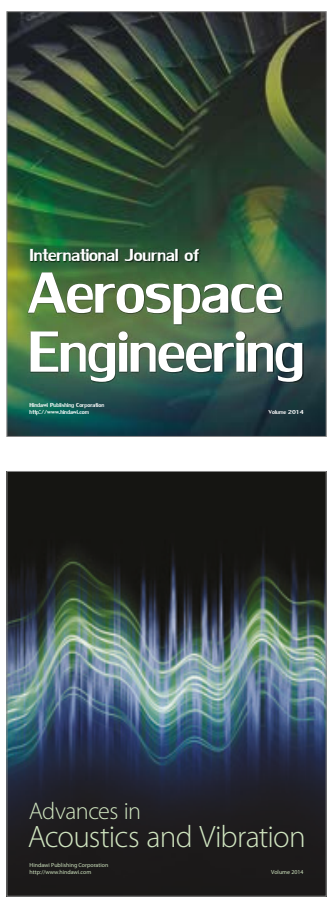

Sensor Networks 